\title{
Coulisses
}

Revue de théâtre

\section{Shakespeare, Richard III}

Mise en scène de Sylvain Meurisse, assisté de Nicolas Laurent. Nouveau Théâtre CDN de Besançon et de Franche-Comté

Claude Peltrault

\section{(2) OpenEdition}

12 Journals

Édition électronique

URL : https://journals.openedition.org/coulisses/809

DOI : $10.4000 /$ coulisses. 809

ISSN : 2546-9460

Éditeur

Presses universitaires de Franche-Comté

\section{Édition imprimée}

Date de publication : 31 décembre 2010

Pagination : 17-21

ISBN : 978-2-84867-302-8

ISSN : $1150-594 \mathrm{X}$

\section{Référence électronique}

Claude Peltrault, "Shakespeare, Richard III », Coulisses [En ligne], 41 | Automne 2010, mis en ligne le 30 novembre 2016, consulté le 02 janvier 2023. URL : http://journals.openedition.org/coulisses/809 ;

DOI : https://doi.org/10.4000/coulisses.809 


\section{Shakespeare, Richard III}

Mise en scène de Sylvain Meurisse, assisté de Nicolas Laurent. Nouveau Théâtre CDN de Besançon et de Franche-Comté

\section{Claude Peltrault}

\section{RÉFÉRENCE}

William Shakespeare, Richard III, mise en scène : Sylvain Meurisse, assisté de Nicolas Laurent. Nouveau Théâtre CDN de Besançon et de Franche-Comté, production : Nouveau Théâtre CDN de Besançon et de Franche-Comté, coproduction : Théâtre Firmin Gémier/La Piscine scène conventionnée d'Antony/Châtenay-Malabry, Théâtre de Sartrouville et des Yvelines - CDN. Spectacle vu le 17 octobre 2009.

1 Avec Richard III, composé au début de la dernière décennie du seizième siècle, le jeune Shakespeare s'intéresse aux ultimes convulsions de la guerre des Deux-Roses, longue période de luttes fratricides pour le pouvoir. Il écrit un drame historique, mais également un chapitre du roman national, et participe à la construction du mythe Tudor, lignée d'où est issue Elizabeth I. Cette entreprise est donc aussi celle d'un poète, qui trouve dans la légende noire d'un Richard contrefait et démoniaque les éléments romanesques propres à faire vibrer l'imagination et la sensibilité du public. Il y a dans le texte assez de lyrisme et de moments pathétiques pour que le spectateur s'attende à passer par toutes sortes d'émotions contradictoires, comme à l'opéra. Coïncidence du calendrier, l'Opéra du Rhin de Strasbourg présentait d'ailleurs, l'automne dernier, le Richard III de Giorgio Battistelli, « dramma per musica », créé en 2005 à Gand. En même temps, le dernier roi Plantagenêt, narcissique et jubilatoire dans la noirceur, a quelque chose du Vice des Moralités et des Interludes, genres en voie d'extinction à l'époque de Shakespeare. On ne peut d'ailleurs nier l'existence d'aspects grotesques dans cette pièce qui ajoute à l'incongruité des situations ou à l'ironie stupéfiante de leur brutal retournement, un bruit et une fureur sanglante parfois proches du grand-guignol.

2 Le spectacle mis en scène par Sylvain Maurice, assisté de Nicolas Laurent, à partir de la traduction de Jean-Michel Déprats, essaie de rendre compte de cette complexité avec toutefois une nette tendance à privilégier la dérision. Le bruit et la fureur sont bien au 
rendez-vous, mais les coupes résultant de la dramaturgie de Denis Loubaton, et la direction d'acteurs, évacuent la plus grande part du lyrisme de la pièce. Il ne s'agit pas ici de contester la liberté de l'équipe artistique, mais c'est un fait. En revanche, il faut saluer la création d'une atmosphère poétique, étrange et poignante, avec l'ajout de ponctuations musicales et vocales (direction Charles Lyet), sortes de mélopées empruntées pour l'essentiel, semble-t-il, à La Nuit du Chasseur de Charles Laughton. Une certaine osmose se produit-elle entre l'univers du film et celui de la pièce, en tout cas, le procédé se révèle particulièrement efficace au moment de suggérer la mort qui rôde autour des jeunes princes dans la Tour.

3 Parmi les points forts du spectacle, il faut souligner l'ingéniosité du dispositif scénique imaginé par François Mercier: un plateau cylindrique entouré d'un anneau, qui peuvent tourner indépendamment l'un de l'autre, et, érigée là-dessus, une structure cylindrique formée de panneaux mobiles. En jouant sur toutes ces possibilités de mouvement circulaire le metteur en scène peut suggérer la dynamique de l'Histoire, l'instabilité de la couronne ou la trajectoire personnelle de personnages emportés dans le tourbillon des luttes de pouvoir, toutes choses symbolisées par la roue de la Fortune. Fermée, cette structure cylindrique présente l'austère silhouette noire de la Tour de Londres où sont exécutés les crimes de Richard. Lorsque les panneaux s'écartent pour les scènes d'intérieur du palais, ils révèlent des parois rougeâtres, comme éclaboussées d'un sang mal lavé. Les spectateurs qui ont vu l'opéra de Battistelli à Strasbourg trouvent ici une variante très réussie de l'arène de sable rouge entourée de gradins imaginée par Mirona Boruzescu, en même temps que les scènes les plus grotesques de Richard et Buckingham peuvent du coup, par association d'idées, faire penser au cirque. Cette double tournette se révèle particulièrement ingénieuse dans les scènes finales, où le noyau central représente la tente du tyran. Pendant la nuit qui précède la bataille de Bosworth, Richard est assailli de cauchemars, l'éclairage projette son ombre sur les parois circulaires, son siège se met à tourner lentement, tandis que le cortège de ses victimes tenant chacune une chandelle allumée, tourne autour de lui comme un manège funèbre de plus en plus rapide. L'ardeur de la bataille est suggérée par une lumière rouge sombre, un vacarme de cuivres, et la folle course bancale de Richard sur le pourtour du plateau toujours en mouvement.

Jean-Baptiste Verquin incarne avec talent un Richard histrionique aux multiples visages. Les spectateurs placés, il se lève en boitant et se présente avec force véhémence et postillons, déjà au moins aussi grotesque que démoniaque. La structure s'ouvre et cette première impression se confirme lorsque Richard réussit le tour de force de séduire Lady Anne - dont il a tué le mari, ainsi que le beau-père, Henry VI devant le cadavre de celui-ci, qui se remet à saigner pour dénoncer son bourreau. C'est dans cette scène difficile, où culmine l'intensité dramatique, qu'est démontrée la fascinante complexité des rapports de haine et de séduction qui unissent Richard à ses victimes, ce qui exige de la part des acteurs beaucoup de présence et une grande subtilité. Á grande attente, grande frustration. Il semble que Lamya Regragui (Anne), souffre d'un léger défaut de prononciation, maîtrisé la plupart du temps, mais qui resurgit ici au pire moment, lorsque s'adressant au cadavre d'Henry VI elle évoque "ton fif affaffiné ", et le débit et le ton de sa malédiction ont aussi quelque chose de faux. Le jeu des deux acteurs se situe essentiellement dans le registre étroit de la véhémence hystérique. 
Lorsque, dans la scène suivante, Margaret vient troubler la réunion de la famille royale, on reste dans le même registre. Bien sûr, Margaret est une furie assoiffée de vengeance mais c'est aussi la solennelle prophétesse des malheurs à venir. Rappelons qu'il s'agit d'une femme mûre, reine en son temps, avec un vécu considérable, qui est réduite à néant par les York, Richard ayant tué son époux et son fils. Ce n'est là que l'effet boomerang de la violence, car les Lancastre aussi ont du sang sur les mains. Catherine Vinatier interprète le rôle avec une énergie qui fait merveille lorsqu'il s'agit de vociférer et de courir dans tous les sens, mais il lui manque quelques années pour rendre parfaitement crédible son personnage de mater dolorosa, et l'étrange perruque filasse dont elle est affublée ne parvient pas tout à fait à faire illusion. Avec Nadine Berland dans le rôle d'Elizabeth, et Murielle Colvez dans celui de la duchesse d'York, la distribution est plus réussie. Elles jouent l'affrontement des deux femmes, vouées l'une comme l'autre à souffrir des crimes de Richard, comme révélateur de leur rivalité dans le concours des malheurs, rivalité qui prend le pas sur la souffrance elle-même. La première exhibe d'abord avec assurance une image paillettes et champagne, qui suggère la jouissance fraîchement acquise du pouvoir, puis on la voit dans la simplicité d'un authentique amour maternel, jouant par terre avec son petit garçon, tout en pressentant « la ruine de sa maison ». Dans la deuxième partie, elle trouve des accents tragiques pour pleurer la mort de ses enfants, rejointe par Murielle Colvez et Catherine Vinatier dans le fameux trio des mères plaintives, mais le grotesque prend vite le dessus : rugissant comme une tigresse, Margaret précipite Elizabeth face contre terre, et avec un rire de démente se moque de son malheur.

6 Philippe Frécon donne au personnage de Clarence ce qu'il faut de naïveté et de tristesse impuissante, c'est un maillon faible, vite expédié par deux tueurs à gages (dont Arnault Lecarpentier). Inaugurant le grand cirque macabre des crimes perpétrés dans la Tour, ils sont interprétés sur le mode clownesque, verts de peur et hilares à l'idée de la récompense, signalés comme appartenant à une engeance intemporelle par un pistolet anachronique. Après la mort d'Edouard, Richard s'en prend à plus faible encore avec l'élimination de ses fils. Le rôle du jeune duc d'York offre à Houda Ben Kamla l'occasion d'une performance remarquable : elle a la voix et la taille de l'enfant et toute la science de l'actrice consommée, ce qui, dans l'échange entre Richard et son neveu, donne au badinage du gamin sur sa petite taille, une résonance particulière.

7 La liquidation de Hastings souligne la volonté du metteur en scène de donner la priorité au grotesque. Comme dans la pièce, on envoie à Hastings un messager en pleine nuit pour le sortir des bras de sa maîtresse, et allez savoir pourquoi, ce messager prend ici l'accent marseillais et y va de son « Oh! Bonneu méreu !» Un ancêtre d'Escartefigue a traversé la Manche! Arrive ensuite Catesby (Lamya Regragui), dans la posture du noceur : semelles à bascule, chapeau pointu et serpentins, bouteille de gin à la main : on transfère sur le personnage ce qu'il dit de l'instabilité du monde. Quant à Vincent Debost, il donne avec justesse au personnage de Hastings la bonhomie d'une force tranquille qui fait de lui la victime idéale de l'ironie dramatique. Dans la scène du Conseil qui précède le couronnement de Richard, les fraises que celui-ci a envoyé cueillir, servent à souligner la naïve confiance du Lord Chamberlain, qui pioche machinalement dans le panier sans se douter le moins du monde de son arrestation imminente, concoctée avec l'aide de Buckingham. C'est Vincent Dissez qui incarne avec brio le rôle de ce dernier, complètement déjanté, séduisant, et trop sûr de son emprise sur Richard auquel il se croit lié par une sorte de fraternité meurtrière. Touchant au 
but, les deux complices, aussi cabotins l'un que l'autre, se laissent aller à une scène de folle exubérance, comme deux frères de défonce shootés au crime. Le chemin du trône étant dégagé, ils envahissent les gradins au rythme endiablé d'une musique sudaméricaine, enjambent les sièges, sortent de leur personnage, de leur texte et de leur époque dans une sorte de délire qui mêle chanson populaire, clins d'oeil aux cousines jurassiennes, réminiscence d'un sketch de Pierre Dac et Francis Blanche etc. Le délire grotesque continue et prend une coloration western lorsque, de retour sur scène, les deux acolytes sèment la terreur en faisant pétarader des pistolets, et toujours armés, se livrent à une danse de sauvages autour de la tête de Hastings que Ratcliffe (Houda Ben Kamla) apporte sur un plateau. Embrassades générales : Ratcliffe offre malicieusement la tête de Hastings au baiser du Lord Maire. La complicité avec la salle se poursuit lorsque Buckingham commentant la froideur du peuple, l'invite à acclamer Richard. Comme de nombreux autres metteurs en scène actuels, Sylvain Maurice exagère ce goût de Shakespeare pour les effets métadramatiques, et multiplie les occasions de faire s'interpénétrer les espaces, les temps et les identités. Deux autres occurrences permettent de le constater, lorsque le gardien de la Tour de Londres joué par Victor Ponomarev, chante à Clarence une berceuse en russe, et lorsque la tunisienne Houda Ben Kamla, dans le rôle de Ratcliffe, chante une mélopée en langue arabe sur le cadavre de Richard.

8 Malgré les quelques faiblesses signalées au cours de ce compte-rendu, c'est un spectateur plutôt heureux de sa soirée qui quitte la salle, la tête encore pleine d'interrogations et d'attentes plus ou moins satisfaites, mais qui ne s'est jamais ennuyé.

Photographie de scène de Richard III, mise en scène de Sylvain Meurisse, assisté de Nicolas Laurent, production du Nouveau Théâtre CDN de Besançon et de Franche-Comté

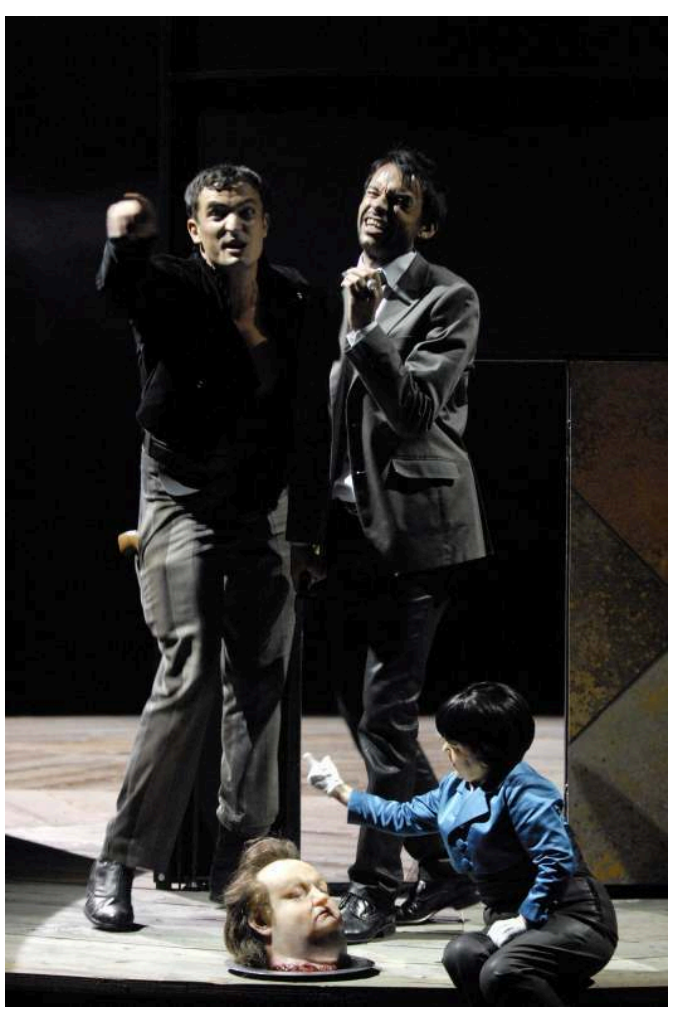

Richard (à gauche) et Buckingham se félicitent de l'exécution de Hastings Elisabeth Carecchio 
INDEX

oeuvrecitee Richard III

Palavras-chave : cenografia

Palabras claves : puesta en escena

Keywords : mise-en-scène

Mots-clés : mise en scène 